九州大学学術情報リポジトリ

Kyushu University Institutional Repository

\title{
INDUCTIVE INFERNCE BY ITERATIVELY WORKING AND CONSISTENT STRATEGIES WITH ANOMALIES
}

Miyahara, Tetsuhiro

Department of Information Systems, Interdisciplinary Graduate School of Engineering Sciences, Kyushu University

https://doi.org/10.5109/13384

出版情報: Bulletin of informatics and cybernetics. 22 (3/4), pp.171-177, 1987-03. Research Association of Statistical Sciences

バージョン :

権利関係 : 


\title{
INDUCTIVE INFERENCE BY ITERATIVELY WORKING AND CONSISTENT STRATEGIES WITH ANOMALIES
}

\author{
By
}

\author{
Tetsuhiro MiYahara*
}

\begin{abstract}
Inductive inference is a process of gaining a general rule from examples. Inductive inference of recursive functions from inputoutput examples is considered. An iteratively working strategy utilizes the last hypothesis produced by it and the present example, and a consistent strategy always produces a program consistent with all examples received so far. An extension of the uniformly bounded number of hypotheses utilized by strategies is shown to lead to no extension of the inferring power. We also show that the technique of $E X^{n}$-hierarchy holds for iteratively working and consistent strategies with anomalies.
\end{abstract}

\section{Introduction}

The present paper deals with the theory of inductive inference which is based on the approaches of Gold $[5,6]$, Blum and Blum [2] and the subject of survey papers (e. g., $[1,10])$. A process of automatic program synthesis can be formalized to inductive inference of recursive functions from examples as an infinite process. Its situation can be imagined as follows: An algorithmic device, which is formally a partial recursive function and called a strategy or an inductive inference machine, takes as the input the graph of a recursive function $f$ which is the list of all examples $(x, f(x))$ for natural number $x$. As it receives the list, it produces infinitely many computer programs called hypotheses. When almost all programs produced by a strategy are equal to a program that computes $f$, we say that the strategy inductively infers (or identifies) $f$. We mean all but finitely many by almost all. A set of recursive functions is said to be identifiable by a strategy if the strategy identifies every function in the set.

There are many possible requirements, called identification criteria, on the process of synthesizing programs and the sequence of programs produced by a strategy. The power of an identification criterion, called an identification type, is expressed by the class of all sets of recursive functions each of which is identifiable by some strategy under the identification criterion. Hierarchies of identification types have been intensively investigated in order to compare the power of the corresponding criteria in inductive inference and automatic program synthesis (e. g., $[3,4,7,9,13]$ ).

In this paper we introduce some new identification criteria and study their powers.

* Department of Information Systems, Interdisciplinary Graduate School of Engineering Sciences, Kyushu University 39, Kasuga 816, Japan. 
Iteratively working strategies, studied in $[7,13]$, are ones which synthesize a program from the last hypothesis they have produced and the present input-output example. We extend them to ones which utilize finite, uniformly bounded, number of hypotheses they have produced and show that their powers are identical to those of the original criteria utilizing only the last hypothesis. Case and Smith [3] have studied almost everywhere identification, introduced in $[2,11]$. Let $n$ be a natural number or *. An anomaly is an input on which a program disagrees with the function being identified. Identification criteria of this type allow the final hypothesis to have $n$ anomalies (finitely many anomalies if $n=*)$. Corresponding identification type is denoted by $E X^{n}$. The relation $E X^{0} \subsetneq E X^{1} \varsubsetneqq \cdots \subsetneq E X^{*}$ is called the anomaly hierarchy or $E X^{n}$-hierarchy [3]. Jantke and Beick [7] have studied natural identification criteria, which requires, for example, that a strategy should produce only programs consistent with examples received so far, and they have clarified the relationship between the power of these natural criteria. We consider some natural identification criteria allowing the hypotheses to have finitely many anomalies and show that the analogous arguments to $E X^{n}$-hierarchy hold for these identification types.

\section{Preliminaries}

We give some basic definitions and notations and present some fundamental results.

$N:=\{0,1,2, \cdots\}$ denotes the set of all natural numbers. The classes of all partial recursive and (total) recursive functions of $n$ variables over $N$ are denoted by $\mathscr{Q}^{n}$ and $\mathscr{R}^{n}$, respectively. For $n=1$ we may omit the superscript indexes. Let $\left(\varphi_{i}\right)_{i \in N}$ be a fixed acceptable numbering of $\mathscr{P}$ [12]. A permutation of $N$ is a complete and repetitionfree sequence of natural numbers. $F(\boldsymbol{N})$ denotes the set of all permutations of $\boldsymbol{N}$. Let $\langle\cdots\rangle$ be a fixed effective encoding of all finite sequences of natural numbers onto $N$. Let $X:=\left(x_{0}, x_{1}, \cdots\right)$ be a sequence of natural numbers, $k, m \in N$ and $m \leqq k+1$. Then we use the notations $X[k]:=\left\langle x_{0}, x_{1}, \cdots, x_{k}\right\rangle$ and

$$
X[k-m+1, k]:= \begin{cases}\left\langle x_{0}, \cdots, x_{k}\right\rangle, & \text { if } k+1<m, \\ \left\langle x_{k-m+1}, \cdots, x_{k}\right\rangle, & \text { otherwise. }\end{cases}
$$

Let $h$ be a sequence of natural numbers, $f \in \mathscr{R}$ and $X \in F(\boldsymbol{N})$. We consider $h, f$ and $f_{\boldsymbol{X}}$ as sequences $\left(h_{0}, h_{1}, \cdots\right),(f(0), f(1), \cdots)$ and $\left(f\left(x_{0}\right), f\left(x_{1}\right), \cdots\right)$, respectively when we use the notations $h[k], f[k], f_{X}[k], h[k-m+1, k], f[k-m+1, k]$ and $f_{X}[k-m+1, k]$.

Let $p, n \in N$ and $f \in R . \quad \varphi_{p}(x) \neq f(x)$ means that $\varphi_{p}(x)$ is defined and not equal to $f(x)$, or $\varphi_{p}(x)$ is undefined. We write $\varphi_{p}={ }^{n} f$ and $\varphi_{p}=* f$ iff $\operatorname{card}\left(\left\{x \in N \mid \varphi_{p}(x) \neq f(x)\right\}\right)$ $\leqq n$ and $\operatorname{card}\left(\left\{x \in N \mid \varphi_{p}(x) \neq f(x)\right\}\right)$ is finite, respectively, where card $(A)$ denotes the cardinality of a set $A$. A sequence $\left(h_{k}\right)_{k \in N}$ of natural numbers is said to converge to a natural number $p$, denoted by $\lim h_{k}=p$, iff almost all elements in the sequence are identical to $p$.

We define the identification type $E X$ which represents the power of the first identification criterion, introduced by Gold $[5,6]$ and called Identification in the Limit. EX coincides with $E X^{0}$ defined above.

DEFINITION 2.1. $U \subseteq \mathscr{R}$ is said to be identifiable in the sense of $E X$ (written $U \in$ 
$E X)$ iff there exists a strategy $S \in \mathcal{P}$ such that $S(f[k])$ is defined, for all $k \in N$ and $f \in U$, the sequence $(S(f[k]))_{k \in N}$ converges to $p$, and $\varphi_{p}=f$.

Thus $E X$ is the class of all sets of recursive functions which are identifiable in the sense of $E X$. Other identification types will be defined similarly. Let $U \subseteq \mathscr{R}$ be identifiable in the sense of $I D$ by a strategy and $f$ belong to $U$. Then we say that the strategy identifies $f$ in the sense of $I D$. Definition 2.2 results from adding the requirement to Definition 2.1 that the strategy has to receive the graph of a function in arbitrary order.

DEFINITION 2.2. $U \subseteq \mathscr{R}$ is said to be identifiable in the sense of $E X^{a r b}$ (written $U \in$ $\left.E X^{a r b}\right)$ iff there exists a strategy $S \in \mathscr{Q}^{2}$ such that $S\left(X[k], f_{X}[k]\right)$ is defined, for all $k \in \boldsymbol{N}$ and $f \in U$ and $X \in F(\boldsymbol{N})$, the sequence $\left(S\left(X[k], f_{X}[k]\right)\right)_{k \in N}$ converges to $p$, and $\varphi_{p}=f$.

Other identification types with the superscript index arb satisfy the similar requirement of the order of graph. Thus we may omit definitions of some identification types with the superscript index arb. Many researchers have considered more restricted or more general identification types than $E X$ and investigated the hierarchies of identification types in detail. By means of Identification by Enumeration [5,6], effectively enumerable sets of recursive functions are identifiable in the sense of $E X$. Thus we also consider here the effective enumerability as a special identification criterion. According to [7] we define

and

$$
N U M !:=\left\{U \leqq R \mid \exists h \in \mathscr{R} ; U=\left\{\varphi_{h(i)} \mid i \in N\right\} \leqq R\right\},
$$

$$
N U M:=\left\{U \subseteq \mathscr{R} \mid \exists h \in \mathscr{R} ; U \subseteq\left\{\varphi_{h(i)} \mid i \in N\right\} \subseteq R\right\} .
$$

The corresponding identification types of iteratively working strategies are denoted by $I T$ and $I T^{a r b}$. Consistent strategy which always produces a program consistent with all examples received so far is very natural. The corresponding identification types are denoted by CONS and CONS ${ }^{a r b}$. In the following theorem we summarize a part of fundamental results of natural identification criteria, which is concerned with this paper.

THEOREM 2.1. (Gold [5], Wiehagen [13], Jantke and Beick [7])

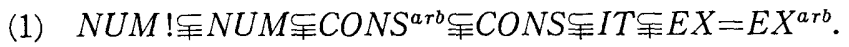

(2) NUM! $\$ I T^{a r b} \lessgtr C O N S$ and $I T^{a r b} \subsetneq I T$,

where $\lessgtr$ denotes incomparability.

We present the theorem describing $E X^{n}$-hierarchy.

DEFINITION 2.3. $U \subseteq \mathscr{R}$ is said to be identifiable in the sense of $E X^{n}(n \in N \cup\{*\})$ (written $\left.U \in E X^{n}\right)$ iff there exists a strategy $S \in \mathscr{P}$ such that $S(f[k])$ is defined, for all $k \in N$ and $f \in U$, the sequence $(S(f[k]))_{k \in N}$ converges to $p$, and $\varphi_{p}={ }^{n} f$.

THEOREM 2.2. (Case and Smith [3]) Let $F^{n}:=\left\{f \in R \mid \varphi_{f(0)}={ }^{n} f\right\}$ for $n \in N \cup\{*\}$. Then the following properties hold:

(1) $F^{k+1} \in E X^{k+1} \backslash E X^{k}$ for all $k \in N$,

(2) $F^{*} \in E X^{*} \backslash \cup_{k \in N} E X^{k}$,

(3) $E X^{0} \varsubsetneqq E X^{1} \varsubsetneqq \cdots \varsubsetneqq \cup_{k \in N} E X^{k} \varsubsetneqq E X^{*}$. 


\section{An Extension of Iteratively Working Strategies}

We extend the iteratively working strategy to the one which synthesizes a program from the last $m$ hypotheses it has produced and the last $n$ examples it has received so far. If it has not yet produced $m$ hypotheses or received $n$ examples, it utilizes all hypotheses or examples. The following identification types represent the power of this postulate.

Definition 3.1. $U \subseteq \mathscr{R}$ is said to be identifiable in the sense of $\operatorname{ITE}_{m, n}(m, n \in \boldsymbol{N})$ (written $U \in I T E_{m, n}$ ) iff there exists a strategy $S \in \mathscr{Q}^{3}$ such that for all $f \in U$

(1) a sequence $\left(h_{k}\right)$ is defined by $h_{0}:=0, h_{1}:=S(h[0], 0, f[0])$ and $h_{k+1}:=S(h[k-m+1, k], k, f[k-n+1, k])(k \geqq 1)$,

(2) $p:=\lim h_{k}$ exists and $\varphi_{p}=f$.

DEFINITION 3.2. $U \subseteq \mathscr{R}$ is said to be identifiable in the sense of $\operatorname{ITE}_{m, n}^{a r b}(m, n \in \boldsymbol{N})$ (written $U \in I T E_{m, n}^{a r b}$ ) iff there exists a strategy $S \in \mathscr{P}^{3}$ such that for all $f \in U$ and all $X:=\left(x_{0}, x_{1}, \cdots\right) \in F(N)$

(1) a sequence $\left(h_{k}\right)$ is defined by $h_{0}:=0, h_{1}:=S\left(h[0], X[0], f_{X}[0]\right)$ and $h_{k+1}:=S\left(h[k-m+1, k], X[k-n+1, k], f_{X}[k-n+1, k]\right)(k \geqq 1)$, and

(2) $p:=\lim h_{k}$ exists and $\varphi_{p}=f$.

The identification types $I T$ and $I T^{a r b}$ coincide with $I T E_{1,1}$ and $I T E_{1,1}^{a r b}$, respectively. Clearly $I T E_{a, c}^{a r b} \subseteq I T E_{b, d}^{a r b}$ and $I T E_{a, c} \subseteq I T E_{b, d}$ if $1 \leqq a \leqq b$ and $1 \leqq c \leqq d$. It is shown that an arbitrary, uniformly bounded, enlargement of the number of hypotheses a strategy may utilize leads to no extension of the corresponding identification types.

THEOREM 3.1. Let $m, n \geqq 1$. Then

(1) $I T E_{1, n}=I T E_{m, n}$,

(2) $I T E_{1, n}^{a r b}=I T E_{m, n}^{a r b}$.

Proof. (1) Clearly it suffices to show that $I T E_{m, n} \subseteq I T E_{1, n}$. We show that for any strategy $S \in \mathscr{Q}^{3}$ there exists a strategy $T \in \mathscr{P}^{3}$ such that $I T E_{m, n}(S) \subseteq I T E_{1, n}(T)$, where $I T E_{m, n}(F)$ denotes the set of all recursive functions that a strategy $F \in \mathscr{Q}^{3}$ identifies in the sense of $I T E_{m, n}$. Since $\left(\varphi_{i}\right)_{i \in N}$ is an acceptable numbering, there exist an injection mapping $c \in \mathbb{R}^{2}$ and $d \in \mathscr{Q}$ such that $\varphi_{c(i, x)}=\varphi_{i}$ and $d(\langle c(i, x)\rangle)=x$ for all $i$ and $x \in N$. Let $S \in \mathscr{Q}^{3}$ be given. We define $T \in \mathscr{P}^{3}$ as follows :

$$
T(x, y, z):= \begin{cases}c(S(x, 0, z),\langle 0, S(x, 0, z)\rangle) & \text { if } y=0 \\ c(S(d(x), y, z), e(d(x), y, z)), & \text { otherwise, }\end{cases}
$$

where $e \in \mathscr{Q}^{3}$ is defined by, for all $j \geqq 1$ and $x_{1}, \cdots, x_{j} \in N$,

$$
e\left(\left\langle x_{1}, \cdots, x_{j}\right\rangle, y, z\right):= \begin{cases}\left\langle x_{1}, \cdots, x_{j}, S\left(\left\langle x_{1}, \cdots, x_{j}\right\rangle, y, z\right)\right\rangle, & \text { if } j<m . \\ \left\langle x_{j+2-m}, \cdots, x_{j}, S\left(\left\langle x_{j+1-m}, \cdots, x_{j}\right\rangle, y, z\right)\right\rangle, & \text { otherwise. }\end{cases}
$$

Let $f \in I T E_{m, n}(S), \quad h_{0}:=0, h_{1}:=S(h[0], 0, f[0])$ and $h_{k+1}:=S(h[k-m+1, k], k, f[k-n$ $+1, k])(k \geqq 1)$. By Definition 3.1, there exist $L \geqq m$ and $H \in \boldsymbol{N}$ such that $h_{k}=H$ for all $k \geqq L$ and $\varphi_{H}=f$. Suppose $i_{0}:=0, i_{1}:=T(h[0], 0, f(0)), i_{k+1}:=T(i[k, k], k, f[k-n+1, k])$ 
$=T\left(\left\langle i_{k}\right\rangle, k, f[k-n+1, k]\right) \quad(k \geqq 1)$. From the definition of $T, i_{k}=c\left(h_{k}, h[k+1-m, k]\right)$ $\langle k \geqq 1)$. Therefore $\lim i_{k}$ exists and equals $c\left(H,\left\langle H^{m}\right\rangle\right)$, where $H^{m}$ denotes the sequence $(H, H, \cdots, H)$ of length $m$. Since $\varphi_{c(H,\langle H} m_{\rangle}=\varphi_{H}=f$, we have $f \in I T E_{1, n}(T)$ and then $I T E_{m, n}(S) \cong I T E_{1, n}(T)$.

(2) Analogous proof holds for (2).

The key idea of the above theorem, suggested by Jantke [8], is as follows. Coding of the information on the last $m$ hypotheses to the present hypothesis and the decoding are effective. The $c$ is the coding function and the $d$ is the decoding function. If converges a sequence of original hypotheses produced by a strategy $S$, so does the sequence of the encoded hypotheses produced by the strategy $T$. Thus the strategy $T$ can simulate the strategy $S$. However this technique, as it is, does not hold for the coding of $n$ examples.

Let $I D$ be an identification type corresponding to an identification criterion. $N U M ! \cap I D$ and $N U M \cap I D$ are considered as special identification types which mean the power of Identification by Enumeration under the criterion. By Theorem 2.1, NUM $\cap I T E_{m, n}=N U M$ and $N U M ! \cap I T E_{m, n}=N U M$ ! for all $m, n \geqq 1$. Clearly $N U M ! \cap I D \cong$ $N U M \cap I D$. Most of identification types $I D$ have the property that if a set of recursive functions is identifiable in the sense of $I D$, then so is any subset of the set. We show that this inclusion is strict if an identification type has this property and contains a set of trivial functions.

THEOREM 3.2.

(1) Let ID be an identification type which has the above property and contains the set of all constant functions. Then

$$
N U M ! \cap I D \varsubsetneqq N U M \cap I D .
$$

(2) Let $m, n \geqq 1$. Then

$$
N U M ! \cap I T E_{m, n}^{a r b} \subsetneq N U M \cap I T E_{m, n}^{a r b} .
$$

Proof. (1) Let $C$ be the set of all constant functions. Then $C$ is countably infinite and $C \in N U M !$. Since $2^{C}$ is uncountable and $N U M !$ is countably infinite, there exists $V_{0} \in 2^{C} \backslash N U M !$. By the property of $I D$, we have $V_{0} \in I D$. Thus $V_{0} \in N U M \cap I D \backslash$ $N U M ! \cap I D$.

(2) Clearly $I T E_{m, n}^{a r b}$ has the property of (1) and contains $C$.

\section{Inductive Inference of Almost Everywhere Correct Programs by Some Naturally Working Strategies}

Now we consider the almost everywhere identification by some naturally working strategies satisfying the postulates for the identification types $I T, I T^{a r b}, C O N S$ and $C O N S^{a r b}$, defined in Section 2. By convention we assume $n \leqq *$ for all $n \in N \cup\{*\}$.

DEFINITION 4.1. $U \subseteq \mathscr{R}$ is said to be identifiable in the sense of $\operatorname{IT}^{n}(n \in \boldsymbol{N} \cup\{*\})$ (written $U \in I T^{n}$ ) iff there exists a strategy $S \in \mathscr{P}^{3}$ such that for all $f \in U$

(1) a sequence $\left(h_{k}\right)$ is defined by $h_{1}:=S(0,0, f(0))$ and $h_{k+1}:=S\left(h_{k}, k, f(k)\right)(k \geqq 1)$, and

(2) $p:=\lim h_{k}$ exists and $\varphi_{p}={ }^{n} f$. 
DEFINITION 4.2. $U \subseteq \mathscr{R}$ is said to be identifiable in the sense of $\operatorname{CONS}^{n}(n \in \boldsymbol{N} \cup\{*\})$ (written $U \in C O N S^{n}$ ) iff there exists a strategy $S \in \mathscr{P}$ such that

(1) $U$ is identifiable in the sense of $E X^{n}$ by $S$, and

(2) for all $f \in U, S$ always produces a program having at most $n$ anomalies (finitely many anomalies if $n=*$ ) with respect to the examples received so far, that is

$$
\operatorname{card}\left(\left\{j \in N \mid j \leqq k, \varphi_{S(f[k])}(j) \neq f(j)\right\}\right) \leqq n \quad \text { for all } k \in N \text {. }
$$

The following identification type CONS-IT ${ }^{a r b, n}$ is the combination [7] of $\operatorname{CONS}^{a r b, n}$ and $I T^{a r b, n}$.

DEFINITION 4.3. $U \subseteq \mathcal{R}$ is said to be identifiable in the sense of CONS-IT ${ }^{a r b, n}$ $\left(n \in \boldsymbol{N} \cup\{*\}\right.$ ) (written $U \in C O N S-I T^{a r b, n}$ ) iff there exists a strategy $S \in \mathscr{Q}^{3}$ such that for all $f \in U$ and all $X:=\left(x_{0}, x_{1}, \cdots\right) \in F(\boldsymbol{N})$

(1) a sequence $\left(h_{k}\right)$ is defined by $h_{1}:=S\left(0, x_{0}, f\left(x_{0}\right)\right)$ and

$$
h_{k+1}:=S\left(h_{k}, x_{k}, f\left(x_{k}\right)\right)(k \geqq 1),
$$

(2) $p:=\lim h_{k}$ exists and $\varphi_{p}={ }^{n} f$, and

(3) $\operatorname{card}\left(\left\{j \in N \mid j<k, \varphi_{h_{k}}\left(x_{j}\right) \neq f\left(x_{j}\right)\right\}\right) \leqq n \quad$ for all $k \geqq 1$.

$I T^{0}, I T^{a r b, 0}, C O N S^{0}, C O N S^{a r b, 0}$ and CONS-IT ${ }^{a r b, 0}$ coincide with IT, IT ${ }^{a r b}, C O N S$, $C O N S^{a r b}$ and CONS-IT ${ }^{a r b}$, respectively. We will see in the proof of Theorem 4.1 and Corollary 4.2 that the technique used in $E X^{n}$-hierarchy [3] is still valid for identification types defined above.

THEOREM 4.1. Let ID $D^{n}$ be identification types and $F^{n}:=\left\{f \in R \mid \varphi_{f(0)}={ }^{n} f\right\}$ for $n \in$ $N \cup\{*\}$. If the following three conditions are satisfied:

(a) $I D^{k} \cong I D^{k+1} \cong I D^{*}$ for all $k \in N$,

(b) $I D^{k} \cong E X^{k}$ for all $k \in N$,

(c) $F^{n} \in I D^{n}$ for all $n \in N \cup\{*\}$,

then the following properties hold:

(1) $I D^{k+1} \nsubseteq E X^{k}$ for all $k \in N$,

(2) $F^{*} \in I D^{*} \backslash \bigcup_{k \in N} I D^{k}$,

(3) $I D^{0} \varsubsetneqq I D^{1} \varsubsetneqq \cdots \varsubsetneqq \bigcup_{k \in N} I D^{k} \varsubsetneqq I D^{*}$. $k \in N$.

Proof. (1) By (1) of Theorem 2.2 and (c), we have $F^{k+1} \in I D^{k+1} \backslash E X^{k}$ for all

(2) Assume that $F^{*} \in \bigcup_{k \in N} I D^{k}$. Then there exists $k \in N$ such that $F^{*} \in I D^{k}$. From $F^{k+1} \subseteq F^{*}$ and (b) it follows that $F^{k+1} \in I D^{k} \subseteq E X^{k}$. This contradicts (1) of Theorem 2.2. Therefore we have $F^{*} \notin \bigcup_{k \in N} I D^{k}$, and then $F^{*} \in I D^{*} \backslash \bigcup_{k \in N} I D^{k}$ from (c).

(3) From (1) of Theorem 2.2 and (b) it follows that $F^{k+1} \notin I D^{k}$ for all $k \in N$. Therefore (a), (c) and (2) show

$$
I D^{0} \varsubsetneqq I D^{1} \varsubsetneqq \cdots \subsetneq \bigcup_{k \in N} I D^{k} \varsubsetneqq I D^{*} .
$$

By Theorem 4.1 we can show that the sequences of identification types $I T^{n}$, $I T^{a r b, n}, C O N S^{n}, C O N S^{a r b, n}$ and CONS-IT ${ }^{a r b, n}$ are strictly increasing in $n$.

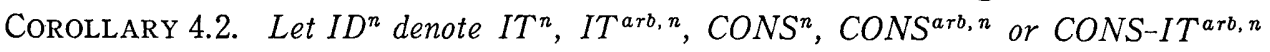
and $F^{n}$ be the set defined in Theorem 4.1. Then for each case the following properties hold: 
(1) $F^{n} \in I D^{n}$ for all $n \in N \cup\{*\}$,

(2) $I D^{k+1} \nsubseteq E X^{k}$ for all $k \in N$,

(3) $F^{*} \in I D^{*} \backslash \bigcup_{k \in N} I D^{k}$,

(4) $I D^{0} \subsetneq I D^{1} \varsubsetneqq \cdots \subsetneq \bigcup_{k \in N} I D^{k} \varsubsetneqq I D^{*}$.

Proof. First of all, we give a proof for the case $I D^{n}=I T^{n}$. (1) Let $S$ be a strategy which produces program $f(0)$ as its only hypothesis when the graph of a recursive function $f$ is fed to it. Formally $S(x, y, z) \in \mathscr{Q}^{3}$ is defined to be equal to $z$ if $y=0, x$ otherwise. Clearly, for all $n \in N \cup\{*\}, F^{n}$ is identifiable in the sense of $I T^{n}$ by $S$.

(2)-(4) By (1) and Definition 4.1, IT ${ }^{n}$ satisfies the conditions (a), (b) and (c) of Theorem 4.1.

By arguments similar to the above case, the properties for the other cases can be proved easily.

\section{Acknowledgements}

The author would like to express his sincere thanks to Prof. Setsuo Arikawa for his constructive comments and encouragements. He is grateful for valuable suggestions from Dr. Makoto Haraguchi. He is also grateful to Dr. Takeshi Shinohara for careful reading and helpful comments.

\section{References}

[1] Angluin, D. and Smith, C.: Inductive Inference: Theory and Methods, Computing Surveys, 15 (1983), 237-269.

[2] Blum, L. and Blum, M.: Toward a Mathematical Theory of Inductive Inference, Information and Control, 28 (1975), 125-155.

[3] CASE, J. and SMITH, C.: Comparison of Identification Criteria for Machine Inductive Inference, Theoretical Computer Science, 25 (1983), 193-220.

[4] CHEN, K.: Tradeoffs in the Inductive Inference of Nearly Minimal Size Programs, Information and Control, 52 (1982), 68-86.

[5] Gold, E. M. : Limiting Recursion, J. Symbolic Logic, 30 (1965), 28-48.

[6] Gold, E. M.: Language Identification in the Limit, Information and Control, 10 (1967), 447-474.

[7] JAntke, K.P. and Beick, H.-R.: Combining Postulates of Naturalness in Inductive Inference, Elektronische Informationsverarbeitung und Kybernetik (EIK), 17 (1981), 465-484.

[8] JANTKE, K.P.: A personal communication (1985).

[9] Kinber, E. B. and Zeugmann, T.: Inductive Inference of Almost Everywhere Correct Programs by Reliably Working Strategies, EIK 21 (1985), 91-100.

[10] KLetTe, R. and Wiehagen, R.: Research in the Theory of Inductive Inference by GDR Mathematicians-A Survey, Information Sciences, 22 (1980), 149-169.

[11] Minicozzi, E. : Some Natural Properties of Strong Identification in Inductive Inference, Theoretical Computer Science, 2 (1976), 345-360.

[12] Rogers, H. JR.: Theory of Recursive Functions and Effective Computability, McGraw-Hill, New York, 1967.

[13] Wienagen, R.: Limes-Erkennung rekursiver Funktionen durch spezielle Strategien, EIK 12 (1976), 93-99.

Communicated by S. Arikawa

Received 'April 14, 1986

Revised October 18, 1986 